\title{
What did we learn about the Milky Way during the last decade, and what shall we learn using Gaia and LSST?
}

\author{
Željko Ivezić ${ }^{1}$, Timothy C. Beers ${ }^{2}$, Mario Jurić ${ }^{3}$, Sarah R. Loebman ${ }^{1}$ \\ and Michael Berry ${ }^{4}$ \\ ${ }^{1}$ Department of Astronomy, University of Washington, Box 351580, Seattle, WA 98195 \\ email: ivezic@astro.washington.edu \\ ${ }^{2}$ National Optical Astronomy Observatory, Tucson, AZ, 85719, and JINA: Joint Institute for \\ Nuclear Astrophysics \\ ${ }^{3}$ LSST Corporation and Steward Observatory, University of Arizona, 933 North Cherry \\ Avenue, Tucson, AZ 85721 \\ ${ }^{4}$ Physics and Astronomy Department, Rutgers University Piscataway, NJ 08854-8019
}

\begin{abstract}
Studies of stellar populations have been reinvigorated during the last decade by the advent of large-area sky surveys such as SDSS, 2MASS, RAVE, and others. These data, together with theoretical and modeling advances, are revolutionizing our understanding of the nature of the Milky Way, and galaxy formation and evolution in general. The abundance of substructure in the multi-dimensional space of various observables, such as position, kinematics, and metallicity, is by now proven beyond doubt, and demonstrates the importance of mergers in the growth of galaxies. Unlike smooth models that involve simple components, the new data reviewed here clearly exhibit many irregular structures, such as the Sagittarius dwarf tidal stream and the Virgo and Pisces overdensities in the halo, and the Monoceros stream closer to the Galactic plane. These recent developments have made it clear that the Milky Way is a complex and dynamic structure, one that is still being shaped by the merging of neighboring smaller galaxies. Here we summarize developments over the last decade in our mapping of the stellar content of the Milky Way, as well as recent attempts to map the dark matter halo by Loebman et al. (2012) and ISM dust distribution by Berry et al. (2012). We also briefly discuss the next generation of wide-field sky surveys, exemplified by Gaia and LSST, which will improve measurement precision manyfold, and comprise billions of individual stars.
\end{abstract}

Keywords. methods: data analysis - stars: statistics - Galaxy: disk, halo, stellar content, structure, interstellar medium

\section{Introduction}

The main purpose of this short review is to summarize some of the recent observational progress in Milky Way studies enabled by SDSS and other surveys, and to extrapolate from them to estimate the performance expected from Gaia and LSST surveys. This contribution certainly does not represent an exhaustive review of all the progress made during the last decade; we refer the reader to much more detailed recent reviews by Ivezić, Beers \& Jurić (2012) and Rix \& Bovy (2013). Indeed, it is difficult to summarize all the exciting progress in the Milky Way studies that happened over the last decade, not only from the observational viewpoint, but including fascinating developments in simulations of galaxy formation and evolution as well.

We first describe the new insights and more reliable quantitative determination of the stellar distribution for the thin disk, thick disk, and halo in the next section, and then 
we discuss recent attempts to map the dark matter halo by Loebman et al. (2012) and ISM dust distribution by Berry et al. (2012) in the following section. A brief discussion of expectations for Gaia and LSST surveys is presented in the last section.

\section{Maps of the stellar distribution in the Milky Way}

The primary driver of the substantial progress in our knowledge of the Milky Way over the last decade is the ability of modern sky surveys to deliver the data required for determining the phase-space coordinates for unprecedented numbers of faint stars over large areas of the sky. In addition to increasing the sample size, the ability to detect faint stars is crucial for extending the sample distance limit. With SDSS, it has become possible to detect even main-sequence (dwarf) stars to a distance limit exceeding $10 \mathrm{kpc}$, and thus to probe both the disk and halo populations within the same dataset. The advantage of carrying out analyses of multiple populations using stellar probes of similar intrinsic properties is difficult to overstate. A theme common to most of the studies reviewed here is the use of photometric parallax relations to estimate stellar distances, followed by the subsequent direct mapping of various distributions using large samples of stars. This mapping approach does not require a-priori model assumptions, and instead constructs multi-dimensional distribution maps first, and only then looks for structure in the maps and compares them to Galactic models.

A key observational breakthrough that made this approach possible was the availability of accurate multi-band optical photometry to a faint flux limit over a large area of sky. The SDSS photometry enabled the development and application of photometric parallax methods, see Ivezić et al. (2008b), which in turn led to direct mapping of stellar distributions in the multi-dimensional space spanned by spatial coordinates, velocity components, and chemical abundance measurements. The resulting maps provided the quantitative basis for separating the main structural components of the Galaxy and obtaining information on their phenomenological description, enabled efficient searches for substructure, and a robust comparison with various model predictions.

\subsection{Separation of the main structural components}

Before the disk and halo can be studied in detail, a robust and accurate scheme for classifying stars into these two components needs to be developed. Using photometric data for $\sim 50$ million stars, Jurić et al. (2008) constructed 3-dimensional maps (data cubes) of the stellar number-density distribution for 19 narrow color bins that span spectral types from mid-F to early M-type stars. When the bin color is varied from the reddest to the bluest one, the maps are "zoomed out", with subsamples covering distances ranging from $100 \mathrm{pc}$ to $15 \mathrm{kpc}$.

These maps are a powerful tool for studying the Milky Way's stellar number density distribution. Traditional methods for modeling stellar counts in the magnitude-color space need to adopt a large number of poorly-known relations, such as the stellar initial mass function, stellar mass-luminosity relationship, stellar luminosity function, and a geometric description of the postulated components such as the disks, bulge, and halo. Alternatively, with these new number-density maps the Milky Way's structure can be examined without any a-priori assumptions about its components: The analysis of the Milky Way's structure is then akin to studies of external galaxies.

The quantitative description of these maps is still a non-trivial task, due to the presence of rich substructures within the components. Nevertheless, the gross behavior can be captured by assuming standard Galaxy models based on two exponential disks and a power-law halo. Jurić et al. (2008) determined the best-fit parameter values for full 

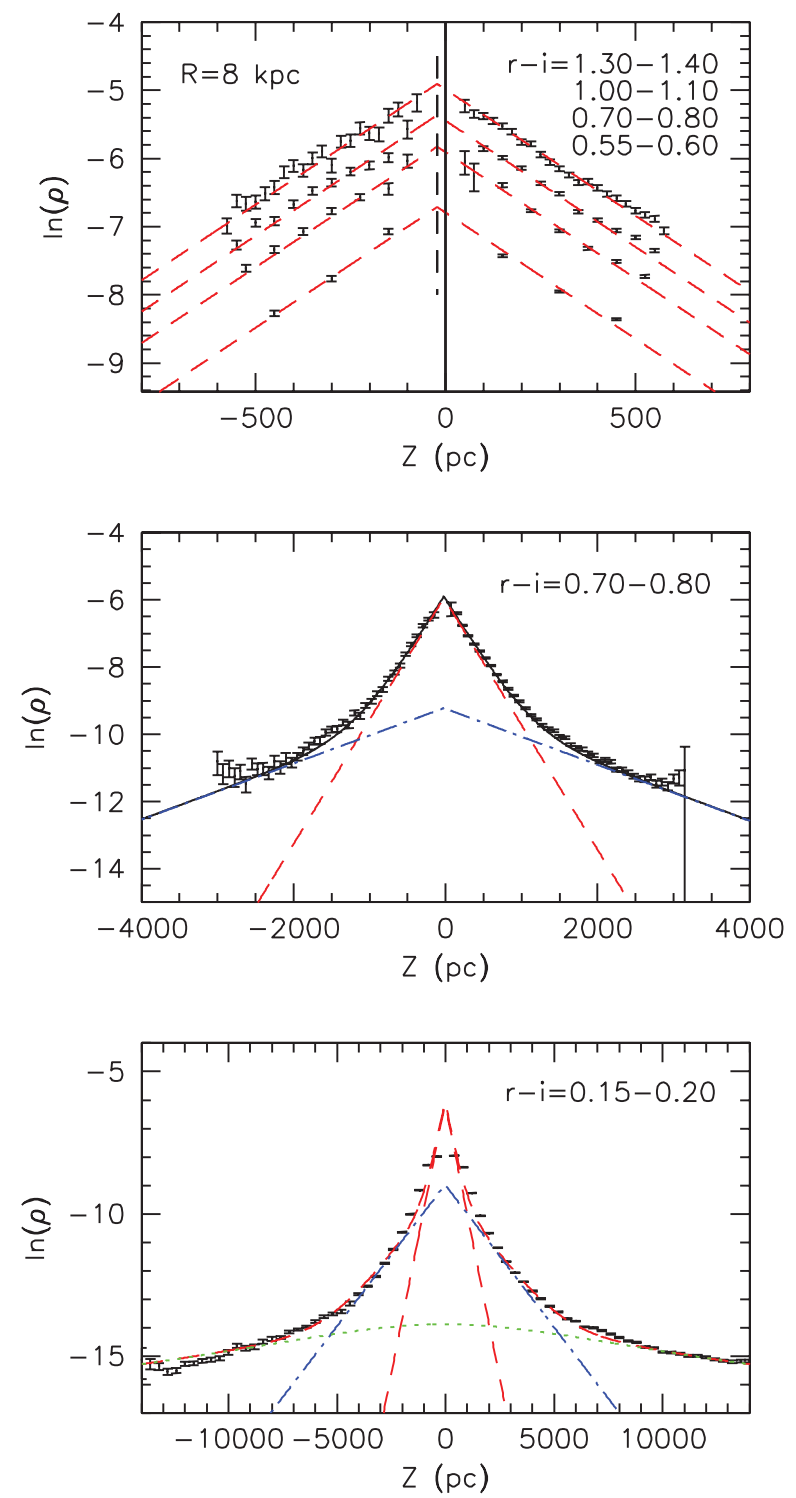

Figure 1. The vertical $(|Z|)$ distribution of stellar number volume density at $R=8 \mathrm{kpc}$ and for different $r-i$ color bins; see Figure 15 from Jurić et al. (2008). The lines are exponential models fitted to the points. The dashed lines in the top panel correspond to a fit with a single, exponential disk. The dashed line in the middle panel corresponds to a sum of two disks with scale heights of $270 \mathrm{pc}$ and $1200 \mathrm{pc}$, respectively, and a relative normalization of 0.04 (the "thin" and the "thick" disks). The dashed line in the bottom panel (closely following the data points) corresponds to a sum of two disks and a power-law spherical halo. The dashed line and the dot-dashed line are the disk contributions, and the halo contribution is shown by the dotted line. For the best-fit parameters see Table 10 in Jurić et al. (2008).

two-dimensional smooth models, and further refined them using residual minimization algorithms; for best-fit parameters, see Table 10 in Jurić et al. (2008).

A cross section of those maps in the direction perpendicular to the disk plane is shown in Figure 1. The data shown in the middle and bottom panels clearly confirm a change in the number-count behavior around $|Z| \sim 1-1.5 \mathrm{kpc}$, usually interpreted as evidence for 

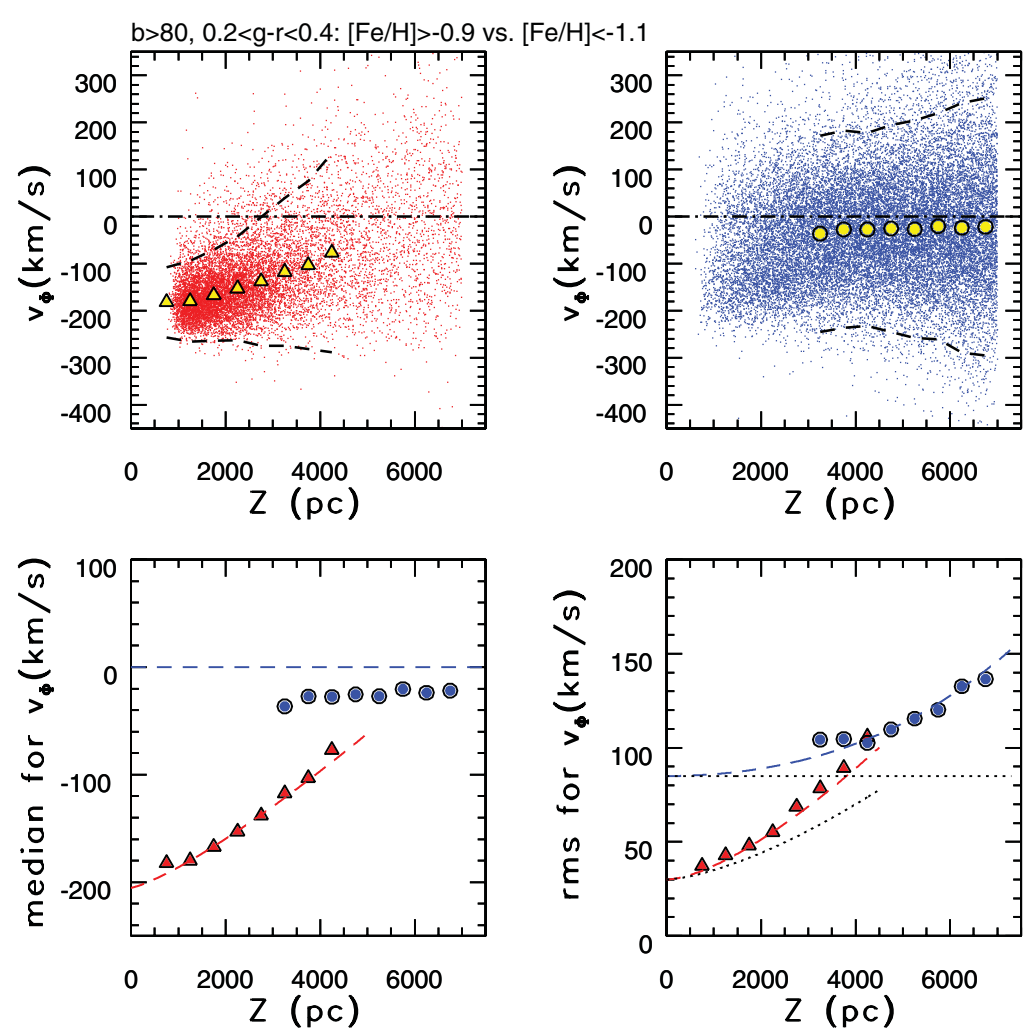

Figure 2. A comparison of the variation of rotational velocity, $v_{\phi}$, on distance from the Galactic plane, $|Z|$, for 14,000 high-metallicity $([\mathrm{Fe} / \mathrm{H}]>-0.9$, top left panel) and 23,000 low-metallicity $\left([\mathrm{Fe} / \mathrm{H}]<-1.1\right.$, top right panel) stars with $|b|>80^{\circ}$; see Figure 15 from Bond et al. (2010). In the top two panels, individual stars are plotted as small dots, and the medians in bins of $|Z|$ are plotted as large symbols. The $2-\sigma$ envelope around the medians is shown by dashed lines. The bottom two panels compare the medians (left) and dispersions (right) for the two subsamples shown in the top panels, and the dashed lines in the bottom two panels show predictions of a kinematic model from Bond et al. (2010). The dotted lines in the bottom right panel show model dispersions without correction for measurement errors.

an extended "thick" disk component. At the point where the additional, more-extended component becomes unable to explain the star counts, around $|Z| \sim 5 \mathrm{kpc}$, another component - the stellar halo - is invoked to explain the data.

Although these modern counts have exceedingly low statistical noise and fairly wellunderstood systematics, the three-component fit to the data shown in the bottom panel begs the question whether a single-component fit with some other function, parametrized with fewer free parameters, might suffice. Detailed analysis of the kinematics and chemistry (parametrized by $[\mathrm{Fe} / \mathrm{H}]$ and $[\alpha / F e]$ ) demonstrates that the adopted components are indeed distinct, and are not due to over-fitting of the stellar counts data.

For example, the halo and disk components have distinct metallicity distributions, and also have vastly different kinematic behavior, as shown in Figure 2. The high-metallicity disk stars exhibit large rotational velocity (about $220 \mathrm{~km} \mathrm{~s}^{-1}$ ), while the low-metallicity halo stars display behavior consistent with no net rotation (to within $10-20 \mathrm{~km} \mathrm{~s}^{-1}$ ). Similar to the behavior of their metallicity distributions, the rotational velocity for disk stars decreases with the distance from the Galactic plane, while it remains constant for 
nearby halo stars. Therefore, reasonably clean subsamples of halo and disk stars can be defined using a simple metallicity boundary $[\mathrm{Fe} / \mathrm{H}]=-1$.

\subsection{A closer look at the thin and thick disks using $[\alpha / F e]$}

Lee et al. (2011) recently analyzed a sample of $\sim 17,000$ G-type dwarfs with $[\alpha / F e]$ measurements from SDSS. This dataset is the first massive sample of stars at distances of several kpc with reasonably accurate distance estimates, measurements of all three velocity components, measurements of both $[\mathrm{Fe} / \mathrm{H}]$ and $[\alpha / \mathrm{Fe}]$, and selected using wellunderstood and simple color and flux selection criteria over a large area of sky.

Thanks to these advantages, the Lee et al. (2011) sample enabled a number of farreaching observational breakthroughs, including a strong motivation for the separation of the sample by a simple $[\alpha / F e]$ cut into two subsamples that closely resemble traditional thin and thick disks in their spatial distributions, $[\mathrm{Fe} / \mathrm{H}]$ distributions, and distributions of their rotational velocity; see Figure 3.

The fact that all three distributions $\left([\alpha / F e],[F e / H]\right.$, and $\left.v_{\Phi}\right)$ in the $|Z|=400-600 \mathrm{pc}$ bin can be described as linear combinations of the corresponding distributions in a distant bin dominated by the thick-disk component and a best-fit thin-disk Gaussian, with the same weights for all three cases that are consistent with the double-exponential fit to the star counts, strongly supports the hypothesis that the Milky Way disk comprises at least two distinct components.

However, the analysis presented in Figure 3 cannot exclude the possibility that the disk structure is more complex than implied by the sum of only two simple components. For
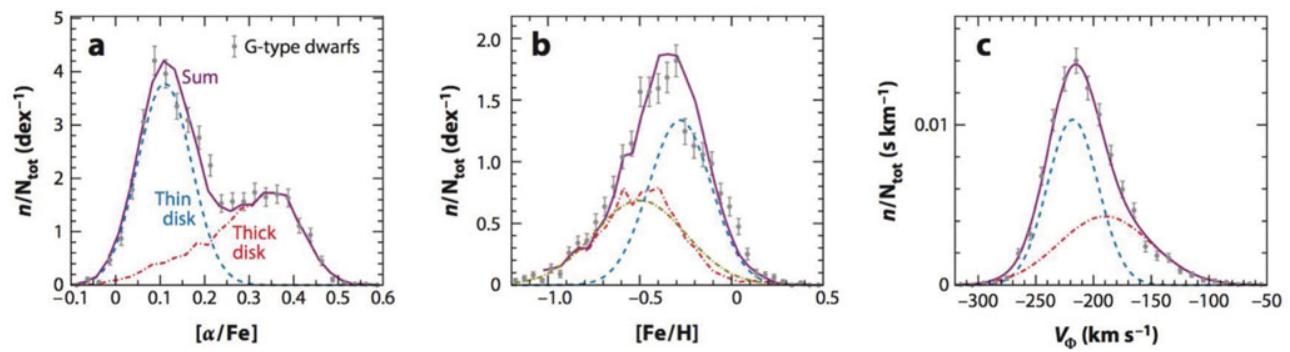

Figure 3. Tests of thin/thick-disk decomposition, using the sample of G-type dwarfs from Lee et al. (2011); see Figure 12 from Ivezić, Beers \& Jurić (2012)). The left panel shows the $[\alpha / F e]$ distribution for $\sim 2,300$ stars in the fiducial bin $|Z|=400-600 \mathrm{pc}$ as symbols with error bars. The bimodality is easily seen. The observed distribution can be modeled as the sum (shown by the magenta solid line) of two components: the $[\alpha / F e]$ distribution for $\sim 3,300$ stars with $|Z|=2-3 \mathrm{kpc}$ shifted to lower values by 0.03 dex (red dot-dashed line) and a Gaussian distribution, $N(0.11,0.06)$ (blue dashed line). The weights for the two components $(0.43$ and 0.57 , for the thick and thin component, respectively) are consistent with a double-exponential fit to star counts. The middle panel shows the $[\mathrm{Fe} / \mathrm{H}]$ distribution for the same stars from the fiducial $Z=400-600 \mathrm{pc}$ bin as symbols with error bars. Similar to the $[\alpha / F e]$ distribution, it can be modeled as the sum (magenta solid line) of two components: the $[\mathrm{Fe} / \mathrm{H}]$ distribution for stars with $|Z|=2-3 \mathrm{kpc}$ shifted to higher values by 0.2 dex (jagged red dot-dashed line) and $N(-0.28,0.17)$ (blue dashed line). The weights for the two components $(0.43$ and 0.57$)$ are the same as in the first panel. The $[\mathrm{Fe} / \mathrm{H}]$ distribution for stars with $|Z|=2-3 \mathrm{kpc}$ is well described by $N(-0.50,0.25)$ (after application of a 0.2 dex offset), shown as the smooth green dot-dashed line. The right panel shows the rotational velocity distribution for the same stars from the fiducial $|Z|=400-600 \mathrm{pc}$ bin as symbols with error bars. It can be modeled as a linear combination of two Gaussian distributions, $N(-218,22)$ and $N(-190,40)$, again using the same relative weights (and line styles) as in the first panel. 
example, Bovy et al. (2012) re-analyzed the same dataset, and concluded that evidence for the bimodal distribution of $[\alpha / F e]$ all but disappears when selection effects are accounted for. The implication of their result is that a continuous distribution of scale heights is a more appropriate model than a simple minimalistic two-component model.

\section{Mapping the invisible stuff}

Observations of visible stars can be used to infer the existence and distribution of the invisible matter - the dark matter halo, through its impact on stellar kinematics, and of the ISM dust, through its reddening of the stellar light (n.b. dust emission can be "seen" at infrared wavelengths). Here we briefly summarize two recent studies that both demonstrated the feasibility of such studies with SDSS data, and illustrated what will be possible with Gaia and LSST data.

\subsection{Can we map the Milky Way dark matter halo?}

Loebman et al. (2012) searched for evidence of dark matter in the Milky Way halo by utilizing the stellar number density distribution and kinematics measured by SDSS, and interpreted by Jeans equations. Because their derived acceleration maps are twodimensional (they assumed cylindrical symmetry), they could focus on the morphology of the resulting acceleration maps, rather than the normalization of the total mass, as done by previous, mostly local, studies; see Bovy \& Tremaine (2012) for a recent measurement of the local dark matter density.

Loebman et al. (2012) found that, in a Newtonian framework, the implied gravitational potential cannot be explained by visible matter alone; see Figure 4 for a quantitative summary. The acceleration experienced by stars at Galactocentric distances of $\sim 20 \mathrm{kpc}$ is as much as three times larger than what can be explained by purely visible matter (using an N-body model). Through application of an analytic method by van der Marel (1991) for measuring the dark matter halo axis ratio, they estimated that SDSS data imply an oblate dark matter halo with an axis ratio of $0.47 \pm 0.14$ within the sampled distance range. These techniques will be used to map the dark matter halo to much larger distances from the Galactic center using upcoming deep optical surveys, such as LSST (see the last section).

\subsection{Can we map the ISM dust distribution?}

At low Galactic latitudes sampled by SDSS SEGUE data, there are severe problems with the interstellar dust extinction corrections. Unlike stars observed at high latitudes, which are predominantly behind the dust layer, stars at low latitudes are embedded in the dust layer. In order to estimate extinction and/or reddening along the line of sight to a star, its distance has to also be estimated.

Berry et al. (2012) used SDSS photometry of 73 million stars to simultaneously constrain the best-fit main-sequence stellar spectral energy distribution (SED) and the amount of dust extinction along the line of sight toward each star. Using a subsample of 23 million stars with 2MASS photometry, whose addition enables more robust results, they showed that SDSS photometry alone is sufficient to break degeneracies between the intrinsic stellar colors and the amount of dust when the shape of extinction curve is fixed; see Figure 5 for an illustration. When using both SDSS and 2MASS photometry, the ratio of the total to selective absorption, $R_{V}$, can be determined with an uncertainty of about 0.1 for most stars in high-extinction regions. Their fits enable detailed studies of the dust properties and its spatial distribution, and of the stellar spatial distribution 

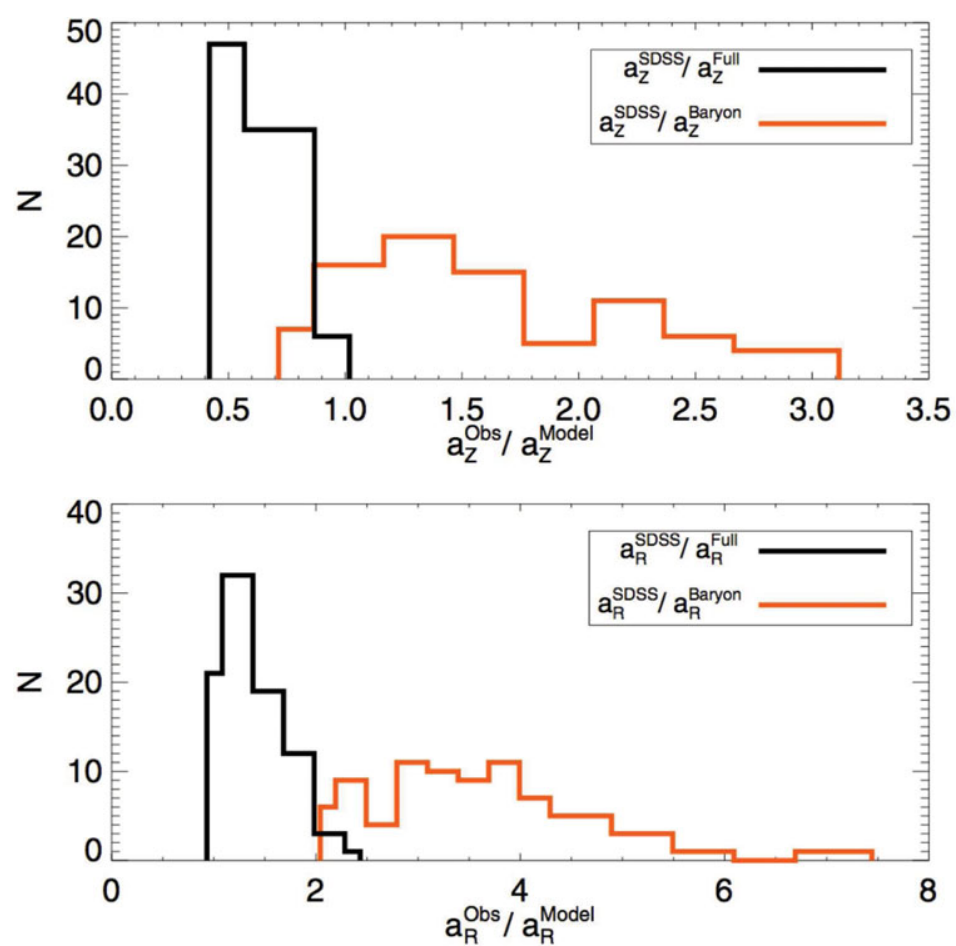

Figure 4. Pixel-by-pixel comparison of the acceleration values implied by the SDSS data and the two model predictions that include (black lines) and do not include (red lines) contributions from dark matter; see Figure 5 from Loebman et al. (2012). The top panel corresponds to the acceleration in the $Z$ direction, and the bottom panel to the $R$ direction; see also Figure 4 from Loebman et al. (2012). The key result is that the model-based acceleration maps that include a dark matter contribution provide a significantly better description of the acceleration maps derived from the SDSS data.

at low Galactic latitudes sampled by SEGUE data†. They analyzed three-dimensional maps of the best-fit $R_{V}$, and found that $R_{V}=3.1$ cannot be ruled out in any of the 10 SEGUE "vertical stripes" at a precision level of $\sim 0.1-0.2$. The best estimate for the intrinsic scatter of $R_{V}$ in the regions probed by SEGUE stripes is $\sim 0.2$.

\section{The Road Ahead}

The last decade has seen fascinating observational progress in Milky Way studies. This progress is expected to continue thanks to multiple large-scale, ground-based projects, including APOGEE, LAMOST, the SkyMapper survey, the Dark Energy Survey, PanSTARRS, and ultimately LSST; for brief technical summaries of these surveys we refer the reader to Ivezić, Beers \& Jurić (2012). These new surveys will extend the faint limit of the current surveys, such as SDSS, by up to 5 magnitudes. In addition, the upcoming Gaia space mission will provide superb astrometric and photometric measurement accuracy for sources with $r<20$, and will enable unprecedented science programs. Here we briefly

$\dagger$ The data and best-fit stellar and dust parameters are publicly available from http://www.astro.washington.edu/users/ivezic/sdss/catalogs/tomoIV/ws.html 

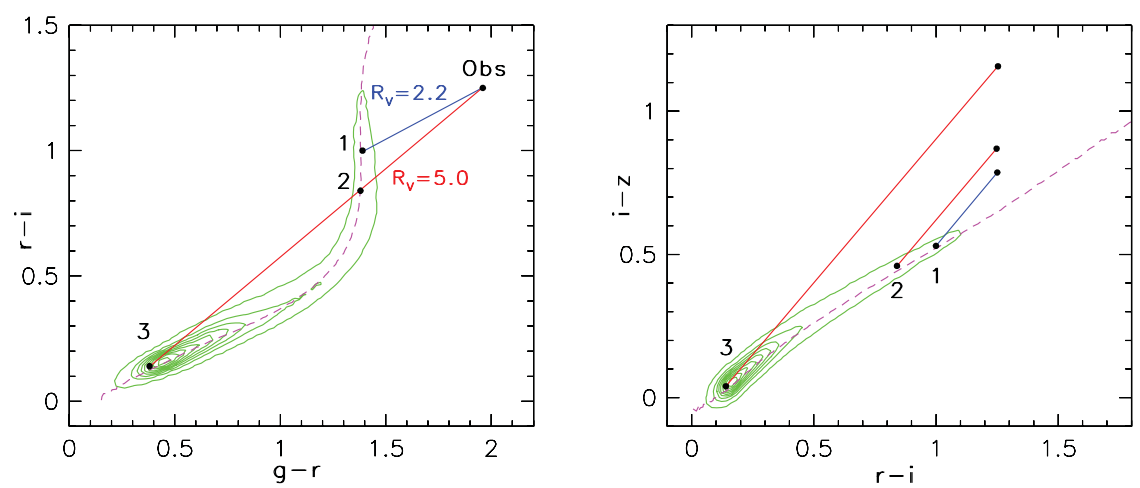

Figure 5. An illustration of the constraints on intrinsic stellar colors, extinction in the $r$ band, $A_{r}$, and the ratio of total to selective extinction, $R_{V}$; see Figure 9 from Berry et al. (2012). In both diagrams, the linearly-spaced contours show the main stellar locus as observed at high Galactic latitudes. The dashed lines mark the median stellar locus from Covey et al. (2010). In the left panel, the dot marked "Obs" represents a hypothetical observation. Depending on the adopted $R_{V}$, as marked, different combinations of intrinsic stellar colors (i.e., the position along the stellar locus) and $A_{r}$ are consistent with the observed $g-r$ and $r-i$ colors. Multiple solutions are possible even for a fixed value of $R_{V}$. The three solutions marked 1-3 correspond to $\left(R_{V}, A_{r}\right)=$ $1:(2.2,1.0), 2:(5.0,2.2)$, and $3:(5.0,6.0)$. As shown in the right panel, these degeneracies are broken if the $i-z$ color is also available: the three $\left(R_{V}, A_{r}\right)$ combinations have different reddened $i-z$ colors, which breaks the degeneracy between the intrinsic stellar color and $A_{r}$. The degeneracy is broken because the reddening vectors in the right panel are nearly parallel despite very different $R_{V}$ values.

compare Gaia and LSST, and discuss the impact they are expected to have on Milky Way studies.

\subsection{The Synergy between Gaia and LSST}

In the context of Gaia, the LSST can be thought of as its deep complement. A detailed comparison of Gaia and LSST performance is given in Figure 6. Gaia will provide an all-sky catalog with unsurpassed trigonometric parallax, proper-motion, and photometric measurements to $r \sim 20$, for about $10^{9}$ stars. LSST will extend this map to $r \sim 27$ over half of the sky, detecting about $10^{10}$ stars. Because of Gaia's superb astrometric and photometric quality, and LSST's significantly deeper reach, the two surveys are highly complementary - Gaia will map the Milky Way's disk with unprecedented detail, and LSST will extend this map all the way to the edge of the known halo and beyond.

For example, stars just below the main-sequence turn-off, with $M_{r}=4.5$, will be detected by Gaia to a distance limit of $\sim 10 \mathrm{kpc}(r<20)$, and to $\sim 100 \mathrm{kpc}$ with LSST'S single-epoch data $(r<24.5)$. For intrinsically faint stars, such as late $\mathrm{M}$ dwarfs, L/T dwarfs, and white dwarfs, the deeper limit of LSST will enable detection and characterization of the halo populations. A star with $M_{r}=15$ will be detectable to a distance limit of 100 pc with Gaia and $\sim 800$ pc with LSST, hence the LSST samples will be about 100 times larger. In addition, for a substantial fraction of red stars with $r>20$, LSST will provide trigonometric parallax measurements accurate to better than $10 \%$. Hence, despite the unprecedented performance of Gaia for $r<20$, LSST will enable major discoveries with its deep $r>20$ sky coverage. At the same time, and in addition to its own discoveries, Gaia will provide excellent astrometric and photometric calibration samples for LSST.

In the context of dark matter halo mapping discussed in section 3.1, Gaia will provide measurements of geometric distances (instead of based on photometric parallax relation!) 

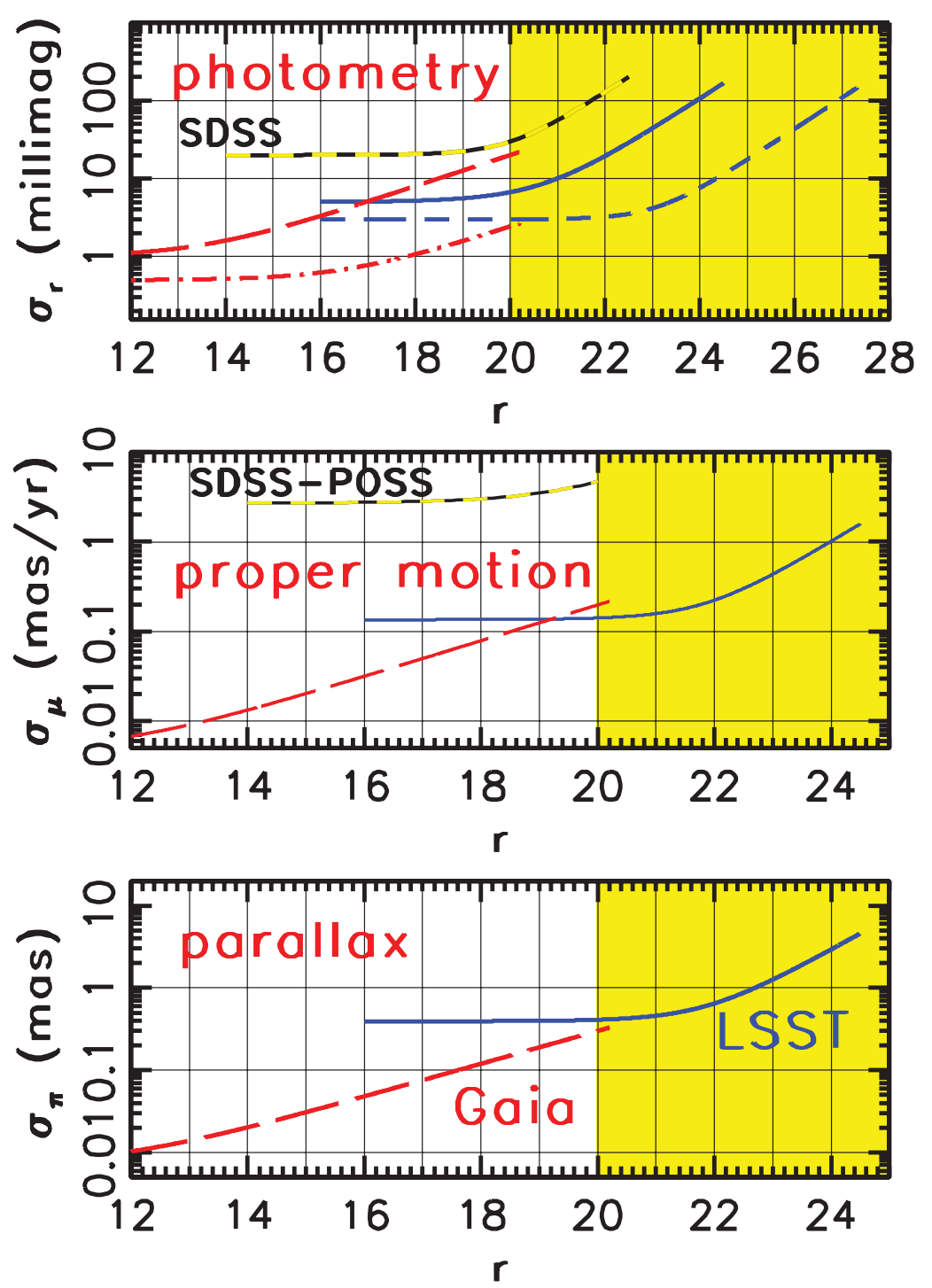

Figure 6. A comparison of the photometric, proper-motion, and parallax errors for SDSS, Gaia, and LSST, as a function of apparent magnitude $r$, for a G2V star; see Figure 21 from Ivezić, Beers \& Jurić (2012). In the top panel, the curve marked "SDSS" corresponds to a single SDSS observation. The red curves correspond to Gaia; the long-dashed curve shows a single-transit accuracy, while the dot-dashed curve shows the end-of-mission accuracy (assuming 70 transits). The blue curves correspond to LSST; the solid curve shows a single -visit accuracy, while the short-dashed curve shows the accuracy for co-added data (assuming 230 visits in the $r$-band). The curve marked "SDSS-POSS" in the middle panel shows the accuracy delivered by the proper-motion catalog of Munn et al. (2004).

and kinematics with a similar faint flux limit as SDSS, but with much smaller errors; see Figure 6. LSST will obtain photometric distances and kinematics of comparable accuracy to those of Gaia at Gaia's faint limit, but extend them deeper by about 5 mag. With Gaia and LSST, it will be possible to extend Galactic gravitational potential studies, such as the one done by Loebman et al. (2012), to distances roughly 10 times larger than 
those possible with SDSS data, revolutionizing our understanding of the Milky Way dark component in the process.

\section{Conclusion}

As briefly illustrated here, both the observational material for studying the Milky Way and the ability of modern simulations to model these observations, have significantly improved during the past decade, and the rapid pace of progress is expected to continue. We conclude by quoting Wyse (2006): "these are exciting times to study local galaxies!" - we couldn't agree more!

\section{References}

Berry, M., Ivezić, Ž., Sesar, B. et al. 2012, ApJ, 757, 166

Bond, B., Ivezić, Ž., Sesar, B. et al. 2010, ApJ, 716, 1

Bovy, J., Rix, H.-W., \& Hogg, D. W. 2012, ApJ, 751, 131

Bovy, J., \& Tremaine, S. 2012, ApJ, 756, 89

Covey, K. R., Ivezić, Ž., Schlegel, D. et al. 2007, AJ, 134, 2398

Ivezić, Ž., Tyson, J. A., Acosta, E. et al. 2008a, arXiv:0805.2366

Ivezić, Ž., Sesar, B., Jurić, M. et al. 2008b, ApJ, 684, 287

Ivezić, Ž., Beers, T. C., \& Jurić, M. 2012, ARAA, 50, 251

Jurić, M., Ivezić, Ž., Brooks, A. et al. 2008, ApJ, 673, 864

Lee, Y. S., Beers, T. C., An, D. et al. 2011, ApJ, 738, 187

Loebman, S. R., Ivezić, Ž., Quinn, T. R., et al. 2012, ApJ, 758, L23

Munn, J. A., Monet, D. G., Levine, S. E. et al. 2004, AJ, 136, 895

Rix, H.-W. \& Bovy, J. 2013, A\& AR, 21, 61

Sesar, B., Ivezić, Ž., Grammer, S. H. et al. 2010, ApJ, 708, 717

van der Marel, R. P. 1991, MNRAS, 248, 515

Wyse, R. F.G. 2006, arXiv:astro-ph/0701832

\section{Discussion}

YANG HUANG: I want to ask a question about the determination of the shape of the dark matter halo by SDSS data. These tracers you show are just several kpc from the plane. They just can constrain the inner-halo shape. So, how to determine the outer-halo shape?

ŽELJKo IVEZIĆ: The stellar acceleration maps from Loebman et al. extend to Galactocentric radius of $20 \mathrm{kpc}$ and thus correspond to the inner halo. To extend this method to the outer halo (say, beyond $30 \mathrm{kpc}$ ), we would need kinematic data to a much fainter magnitude limit than delivered by the SDSS-POSS proper motion catalog, such as expected from LSST, or much more precise kinematic data at the same depth, so that we can apply this method to distant luminous giant stars, such as expected from Gaia. Nevertheless, there is already convincing published evidence that the radial velocity dispersion for halo stars decreases between $30 \mathrm{kpc}$ and $100 \mathrm{kpc}$. This decrease implies that the SDSS-based results for the inner halo cannot be readily extended to the outer halo, because Loebman et al. results are fundamentally based on the SDSS discovery that the halo velocity dispersion expressed in spherical coordinates is invariant at Galactocentric distances below $20 \mathrm{kpc}$.

Johannes Andersen: The scientific output of the LSST will of course be overwhelming. But the same is said about the SKA, ALMA, the E-ELT and TMT, the need for 
spectroscopic followup to Gaia, LSST, and other surveys. Where will the people (and the money) come from in the real world.

ŽELJKO IVEZIĆ: This is a very important question that the world-wide astronomical community is facing now. Let me start by pointing out that the science deliverables promised by LSST do not require extensive spectroscopic followup. Some spectroscopy will be required for calibration purposes, for example to calibrate photometric redshifts of galaxies and photometric metallicity of stars, and the U.S. community has just started a discussion of what resources are needed and how they compare to existing sources (see the recent meeting "Spectroscopy in the Era of LSST", Tucson, April 11-12, 2013). That said, LSST could and should be viewed as an integral part of the overall optical/IR system portfolio. It goes without saying that the scientific output of LSST would be greatly enhanced by commensurate spectroscopic and other follow-up efforts. In the era of scarce funding sources, as well as the increasing costs of our toys, we need to carefully and rationally prioritize what we want to do, and perhaps make extensive use of the world-wide synergistic opportunities. 